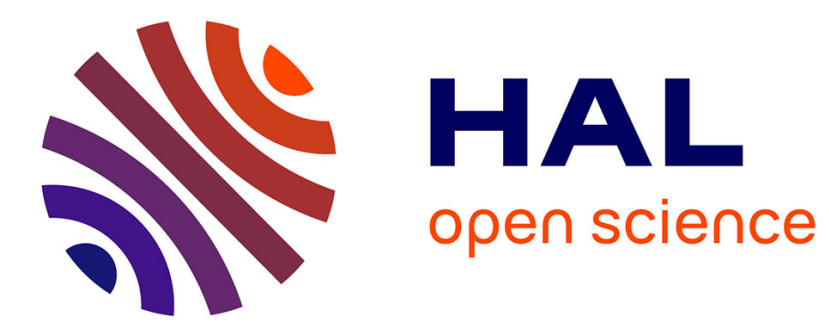

\title{
A methodology to design kinematics of fixations between an orthosis and a human member
}

\author{
Nathanael Jarrasse, Guillaume Morel
}

\section{To cite this version:}

Nathanael Jarrasse, Guillaume Morel. A methodology to design kinematics of fixations between an orthosis and a human member. 2009 IEEE/ASME International Conference on Advanced Intelligent Mechatronics (AIM), Jul 2009, Singapore, Singapore. pp.1958-1963. hal-02110715

\section{HAL Id: hal-02110715 \\ https://hal.science/hal-02110715}

Submitted on 25 Apr 2019

HAL is a multi-disciplinary open access archive for the deposit and dissemination of scientific research documents, whether they are published or not. The documents may come from teaching and research institutions in France or abroad, or from public or private research centers.
L'archive ouverte pluridisciplinaire HAL, est destinée au dépôt et à la diffusion de documents scientifiques de niveau recherche, publiés ou non, émanant des établissements d'enseignement et de recherche français ou étrangers, des laboratoires publics ou privés. 


\title{
A methodology to design kinematics of fixations between an orthosis and a human member
}

\author{
Nathanaël Jarrassé and Guillaume Morel
}

\begin{abstract}
The design of robotic orthoses focuses strongly on replicating kinematics of human limb. However, often sophisticated mechanisms which attempt at reproducing complex kinematics of human joints fails in adapting to geometrical variations of subjects sizes and eccentricities. One major that arrises from this mismatching is an occurrence of hyperstaticity induced by the uncontrolled interaction forces.

In this paper, we take the point of view of statics to investigate the force transmission problem, which is required for a fine force control. The main result of this study focuses on designing fixations between the orthosis and the human limb that provide additional degrees of freedom. The method involves two steps. Firstly, a set of possible solutions with respect to the isostaticity criterion is derived. Then, among these possible solutions, a set of design rules considering physiological aspects of transmitting forces to human limbs is used to select a preferred configuration. As an example, the method is applied to an existing 4 active DOF arm orthosis.
\end{abstract}

\section{INTRODUCTION}

Whatever the particular use they may be designed for (boosting human force capabilities, helping a patient during a neurophysical rehabilitation, etc.), the major purpose of exoskeletons is to transmit forces to the connected human limb. Designing these physically connected devices faces a rather challenging set of constraints: adaptability to kinematics variations between human subjects is required; a large force capability is desirable over a large workspace; simultaneously transparency (i.e. capability of applying minimal forces in resistance to the subject's movements) is of high importance. Indeed, the device should not disturb the intentional motion of the subject during all the phases where assistance is not required [1], which is of critical importance in a rehabilitation program where patients suffer from low motor capabilities [2]. These characteristics are often hard to conciliate within a single device.

For several years, researchers have worked on the primary technical problems raised by these challenges: actuators, power transmission, embedded energy. The first results of these works are remarkable: new actuators [3] or embedded technologies[4] came out of the rich design activity in this domain (for an overview, see [5]). Active powered exoskeletons are now available for rehabilitation [6], for military applications [7] or even for assisting construction workers [8]. Designing the kinematics of an exoskeleton consists of trying to replicate the human limb kinematics. This brings a number of advantages: similarity of the workspaces, singularity

N. Jarrassé and G. Morel are with University P. et M. Curie, ISIR (Institut des Systèmes Intelligents et de Robotique) CNRS - UMR 7222)). 4 place jussieu, 75005 Paris - France. Telephone: +33.1.44.27.51.41

jarrasse@isir.fr, morel@upmc.fr avoidance [9], natural feeling of the connection with human subject. If the kinematics of the human limb and the orthosis are the same, there is a one-to-one mapping between the joint torques exerted by the robot and the joint torques applied to the human subject, whatever the joint configuration. This allows to avoid force transmission variability over the workspace that is observed when, for example, two separate external mechanisms are connected to the two segments of an arm, [10].

A major drawback of the orthosis paradigm is that, in fact, human kinematics is impossible to replicate with a robot. Two problems occurs: morphology drastically varies from a subject to another and, for a given subject, the joints kinematics is very complex and can not be imitated by conventional robot joints [11]. In fact, it is impossible to find any consensual model of the human kinematics in the biomechanics literature. Human joint movements result from the complex geometry of bones interacting surfaces, with a relative bone motion produced through a complex set of mechanically distributed actuators. They are difficult to identify non invasively, since only the motion of the skin can be directly observed. For example, human arm structure is very hard to reproduce: different models exist for the group shoulder-scapula-clavicle, with at least 5 DOF [12]; the elbow joint, often modeled by a simple pivot, is in fact close to an helical axis rotation joint; the localization and nature of the prono-supination joint vary from a model to another.

All these unavoidable modeling uncertainties for the human limbs lead to approximate models and then to imperfect exoskeleton copies, which in turn generates kinematic compatibility problems. Indeed, when connecting two-by-two the links of two kinematically similar chains that are not perfectly identical, hyperstaticity occur which results, if rigid models are used, to the impossibility of moving and the apparition of non controllable (possibly infinite) internal forces. In practice, though, rigidity is not infinite and assembly mobility can be obtained thanks to deformations. When a robotic orthosis and a human limb are connected, most likely, these deformations occur at the interface between the two kinematic chains, thanks to the low stiffness of skin, tissues, and eventually additional compliance provided at the fixations level by using elastic straps or pneumatic systems [13]. Additionally, in some cases, unmodeled redundancy of the human limb structure helps reducing the hyperstaticity degree. Deformations induced at the interface level are thus reduced thanks to an internal motion of the patient, but this motion is uncontrolled and may be undesirable, e.g. in rehabilitation applications. For example, the majority of existing arm exoskeletons possesses a ball 
joint shoulder, while the human glenohumeral joint center is not fixed. If hyperstaticity results from the fixation of the orthosis to the arm, most likely, the human subject will use the motion of the scapula to adapt the glenohumeral center position in such a way that interaction forces will be limited. In a rehabilitation system, this may be drastically inappropriate. In order to quantify forces which can occur from the discrepancy between robot and human kinematics, we presented in Fig. 1 the result of a simple simulation case, where physical and geometrical parameters where chosen to be of the same order of a real situation. Based on the work published in [14] to study a 1 DOF problem, we compute forces generated by constraints and deformations when connecting two 2 DOF chains with the same kinematic structures but with little error in the axis alignment and members length. Two fixations are considered. The deformations in the model are localized at the fixations level thanks to springs which stiffness was chosen from the estimation of the limb tissues stiffness in [14]. Figure 1 presents the simplified kinematic model and a force level graph for the cnemis fixation for angular variation at the hips $\beta_{1}$ and cnemis $\beta_{2}$ with a $2 \mathrm{~cm}$ positioning misalignment between the orthosis and leg axes.
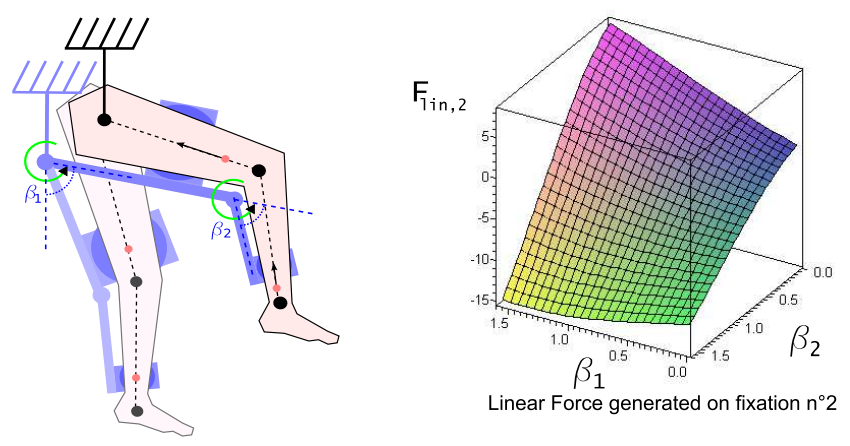

Fig. 1. Model of constraints forces appearance due to model error and hyperstaticity in the cnemis fixation

The maximal amount of force applied at the fixation is about $15 \mathrm{~N}$ and thus cannot be neglected. To cope with this problem, several studies have been conducted in order to improve the orthoses structure and the interaction quality: robotic segments with adjustable length were developed, pneumatic systems were added to introduce elasticity in the robot fixations and adaptability to variant limb section [13]. Another way of thinking the design of orthosis, is to create serial structure not inspired by the human limbs. The recommended solution is to add degree of freedom (active or passive) inside the chain between the active joint matched to human ones [15]. These added DOF in the chain are mainly used to overcome the misalignment problems of rotations axis, and create self aligning structures. This drastically increases the structure complexity and the control problems. In [13], a new design approach to create ergonomic orthotic devices is proposed. It consists of a set of rules that should be respected during the design. A 9 DOF ergonomic orthosis, adaptable to morphological variations and offering a large workspace is proposed.
However, this device is passive and the force transmission capability is not within the scope of the study.

Adding passive DOF between the robot and the human limb, or increasing the adaptability of the robot geometry are parts of the solution but a careful consideration of the force transmission problem for active orthoses is missing in the literature to our knowledge. In this paper, we consider a generic problem of coupling an orthosis with a human limb by adding passive DOF at the interface between the structures. We provide design criteria allowing to solve the problem of morphology compatibility, misalignment errors but also force transmission by removing hyperstaticity. As a result, the design method can be associated with simple force control, where the number of independent forces to be controlled, distributed over the human limb, equals the number of orthosis actuator.

The paper shows in Section II the general design approach. Then, in Section III, the general method is applied to ABLE, a 4 active DOF arm orthosis, comparing different possible solutions for isostatic coupling with a human arm.

\section{General Methodology}

The main question addressed in this paper can be defined as follows: given a proposed orthotic structure with the fixed geometric parameters, conceived to replicate a human limb kinematic model, how to connect it to the human limb while avoiding uncontrollable forces at the interface, even in the case of discrepancies between the model and the real arm kinematics? The answer takes the form of a set of passive frictionless mechanisms used to connect the robot and the subject's limb that allows to avoid hyperstaticity.

\section{A. Removing hyperstaticity}

We consider two different serial chains with multiple couplings as illustrated in Fig. 2. One is representing an human limb $\mathbf{H}$ and the other the robot structure $\mathbf{R}$.

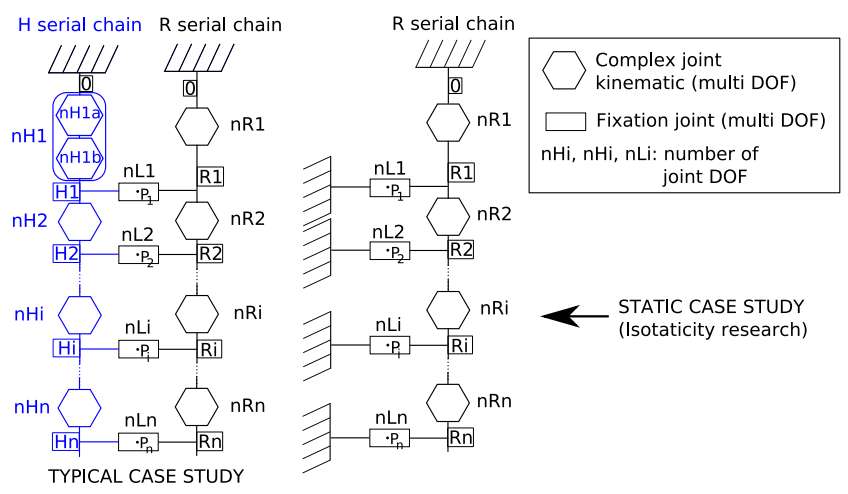

Fig. 2. Schematic of two serial chains parallel coupling

The human limb is supposed to be composed of $n$ segments, each of them being connected to a robot link; $\mathbf{n} \mathbf{L}_{i}$ represents the number of DOF of the $i^{t h}$ fixation between the two chains, while $\mathbf{n} \mathbf{R}_{i}$ represents the number of active DOF of the robotic mechanism (joint) between the $(i-1)^{t h}$ and $t^{t h}$ fixation, where the fixation 0 is the rigid embedment between the two chain 
bases. In order to study the hyperstaticity of the system, we shall consider a fixed human limb with infinite rigidity, as illustrated on the right part of Figure 2. Each of the $n$ embedments provides 6 static unknowns, while each of the $n$ robot active joints allows to control $\mathbf{n R}_{i}$ variables. Therefore, if we avoid singular configurations, which will be supposed in the rest of the paper, the total number of free movements that should be added to provide isostaticity is:

$$
\sum_{i=1}^{n} \mathbf{n L}_{i}=6 n-\sum_{i=1}^{n} \mathbf{n} \mathbf{R}_{i}
$$

In order to determine how to distribute the additional passive mobilities among the fixations, one further consider the problem in an iterative way, considering first the proximal fixation as illustrated in Figure 3. More precisely, we consider the

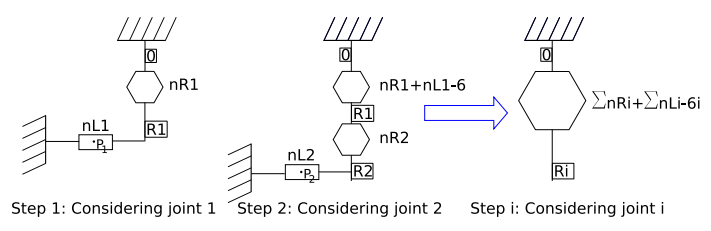

Fig. 3. Robot serial chain study and iterative calculation

closed loop chain constituted by the base 0 , the first robot joint and the first fixation. This sole loop is not required to be isostatic, since it is connected through the next limbs to other kinematic constraints. Rather, we only need to ensure that this loop is not hyperstatic, namely:

$$
\mathbf{n L}_{1} \geq 6-\mathbf{n} \mathbf{R}_{1}
$$

If no kinematic constraint were added through the rest of the orthosis, then the mobility of the first closed loop chain would be $\mathbf{m}_{1}=\mathbf{n L}_{1}+\mathbf{n} \mathbf{R}_{1}-6$. In this case, the hyperstaticity avoidance constraint for the second chain would write $\mathbf{n L}_{2} \geq 6-\mathbf{n R}_{2}-\mathbf{m}_{1}$. Generalizing for the $i^{\text {th }}$ fixation, we have:

$$
\mathbf{n L}_{i} \geq 6-\left(\mathbf{n R}_{i}+\mathbf{m}_{i-1}\right)
$$

with

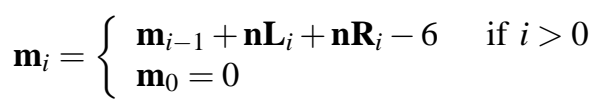

Note again that if only fixations 1 to $i$ are placed, a positive mobility (uncontrolled movement) can be obtained for the $i^{\text {th }}$ body. However, according to Eq. 1, it is guaranteed that the overall system is isostatic; thus, it does not move. Following these last equations, we are able to calculate iteratively the different options for distributing the additional passive DOF at fixations over the structure. We can build a tree of all possible solutions for $\mathbf{n L}_{i}$. In order to choose among these possible set of solutions, we developed a set of rules considering the particularities generated by the fact that forces and moments are being applied to a human limb.

\section{B. Choosing appropriate passive DOF for the fixations}

We propose now a set of design rules more dedicated to fine human-robot interaction. We have in mind applications like arm robotic rehabilitation of an hemiparetic patient, where sensitivity and pain are exacerbated and where a fine control of small interaction forces is necessary. These rules are mainly governed by physiological reasons.

Rule 1. Instead of moments at the fixations points only pure forces should be transmitted.

Here, what we call the fixation point is the point $P_{i}$ where the wrench expressing the interaction between the robot and the human at the $i^{\text {th }}$ fixation will be expressed. Roughly representing the $i^{t h}$ human limb by a straight line $D_{i}, P_{i}$ is to be chosen belonging to $D_{i}$, see rule 4 . Transmitting moments at a fixation point is undesirable for two reasons. First, transmitting a torsion around $D_{i}$ would generate large deformations of the muscles thus involving a large fiber elongation.

Also, applying directly this moment through a tight fixation is in fact a transmission by friction that can generate high tangential forces on the skin, and thus, pain. As illustrated in Fig. 4, it is often possible to use a couple of forces applied to two segments in order to create a torque around a limb axis. In terms of local deformations of the skin and muscles, it is highly preferable. Note that the solution sketched in Fig. 4 is

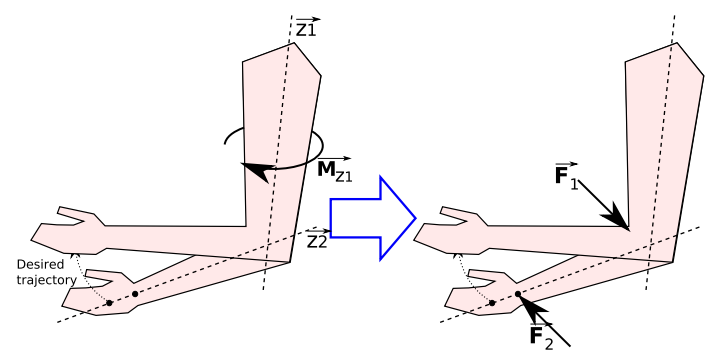

Fig. 4. Illustration of rule 1 : Splitted forces instead of torques

not possible at full extension, where the two segment axes are aligned, but, again, singularities are supposed to be avoided in this paper.

Transmitting a moment around an axis perpendicular to $D_{i}$ is also to be avoided. As illustrated in Figure 5, applying such

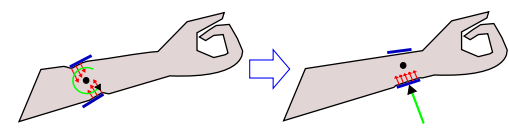

Fig. 5. Illustration of rule 1: Tissue deformation and pressure are less important with pure forces transmission than with moments

a moment results in the concentration at two opposite points of the stress applied to the limb tissues. The local forces may be rather high since the dimensions of the parts in contact with the limb shall remain small for ergonomy purposes. 
Rule 2. Forces need to be transmitted perpendicularly to the limb axis.

Reciprocally, a passive degree of freedom should be added along $D_{i}$. This is simply due to the fact that the human body structure is made of ball-joints and segments, and so that translations along $D_{i}$ directions are not among the possible movements to be assisted.

Rule 3. Setting $\mathbf{n L}_{i}=6$ should be avoided.

The main reason is the will to copy human actuators distributed architecture and thus to split fixation points all along the human limb (see Figure 6). The goal is to favor collocation of the robot actuator and the human joint in order to ease, e.g. force control. The interaction quality level will be enhanced if we limit the stiffness loss due to important displacement of strain through soft tissues chains and control loss due to high number of actuated DOF leading to singularity.

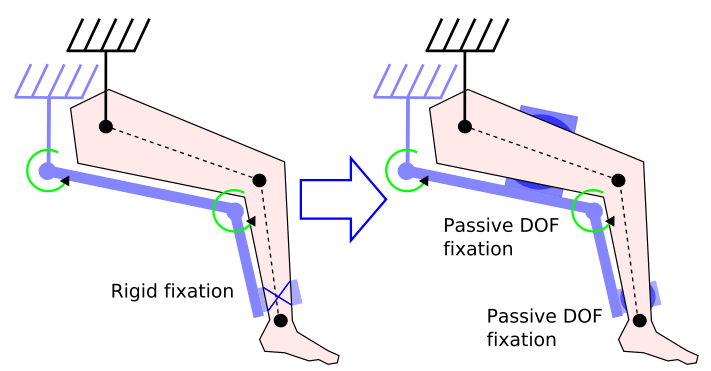

Fig. 6. Illustration of rule 3 : Distributed fixations allow a better interaction quality level

Rule 4. Interaction surfaces need to be large on the human body, outspreaded one from each other and positioned on dimmed sensitive areas.

Fourth and last rule concerns the limitation of contact pressure by interfacing large surface instead of small contact points. Some considerations for the positioning along the limb segments of the fixations should also be added. In order to maximize the force transmission from robot to human, high stiffness areas with low sensitivity tissue (feebly innervated). Several studies have been done on localizing these specific human body areas. For example, on the arm, the wrist is a good place to fix a robot and limit discomfort([9]). Fixations should also be fitted with high density foam insertion or inflatable devices to provide adaptation capabilities to the varying section of the limbs during movement. Moreover, one should also maintain length large enough between two fixations, in order to allow the robot to generate torques along $D_{i}$ with limited forces (see Figure 4).

\section{ApPlication to THE ORTHOSIS ABLE}

\section{A. Presentation of the ABLE upper limb orthosis}

ABLE (see Figure 7) is a 4 axis orthosis that has been designed by CEA-LIST on the basis of an innovative actuation technology ([16]). Its kinematics is composed of a shoulder

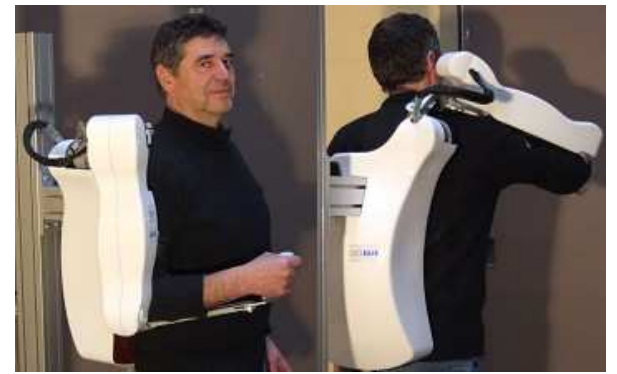

Fig. 7. ABLE 4 axis exoskeleton actuated by screw-and-cable actuators

spherical arrangement made with 3 coincident axes and an elbow. The forearm, terminated by a handle, is not actuated. Its kinematics is sketched in Figure 8. Most of the technological originality of ABLE comes from its actuation and transmission system, which is based on a patented Screw-and-Cable system (SCS) [3]. The hardware characteristics of ABLE make it an excellent platform for physical rehabilitation therapies. Its low joint stiffness and naturally compliant joints ensure the safety when using the robot for patients with physical disability. Unfortunately, first experiments shows us that without paying attention to the fixations by simply connecting arm and forearm middle areas to the orthosis thanks medical straps, which induces hyperstaticity, a clear natural movement alteration appears. This alteration is mainly due to a desynchronization phenomenon between the arm joints: the synergies seems to be perturbed even with an important measured transparency (low inertia and friction phenomenons) and felt by subjects.

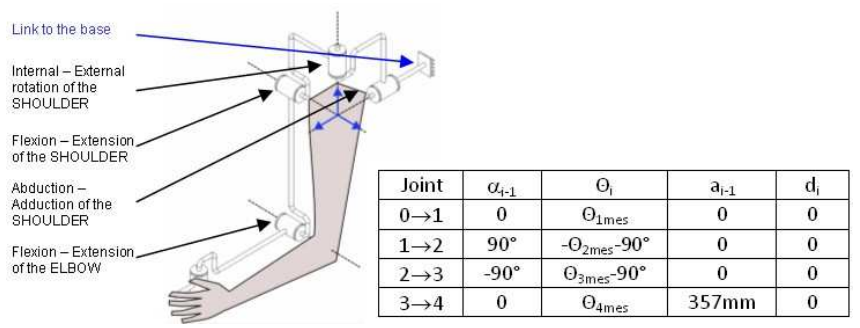

Fig. 8. Kinematics of ABLE

\section{B. Application of our methodology}

To avoid the problem partly due to the fixations in our evaluations, we apply the proposed approach to this particular structure. Firstly, rather than controlling the human arm through only one fixation point, rule 3 advise us to fix every human segment to a robot one, in order to distribute the forces along the arm. Thus we choose to study a two fixations system (See Fig 9). The total number of passive degrees of freedom to be added is:

$$
\sum_{i=1}^{2} \mathbf{n L}_{i}=12-\sum_{i=1}^{2} \mathbf{n} \mathbf{R}_{i}=12-(3+1)=8
$$

The hyperstaticity avoidance constraint is, for the first fixation:

$$
\mathbf{n L}_{1} \geq 6-\mathbf{n R}_{1} \quad \Rightarrow \quad \mathbf{n L}_{1} \geq 3 \text {. }
$$



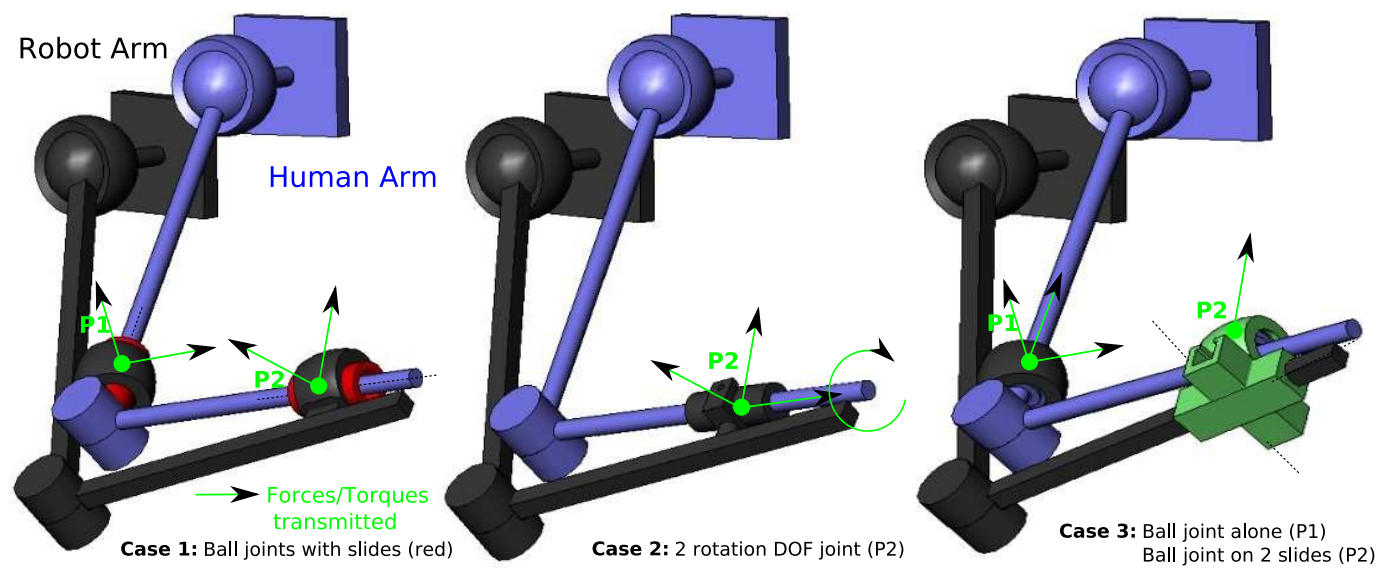

Fig. 10. Schematic of possibilities given by the solution tree for coupling ABLE to an human arm. From left to right: Case $1(\mathbf{n L} 1=4, \mathbf{n L} 2=4)$, Case 2 $(\mathbf{n L} 1=6, \mathbf{n L} 2=2)$, Case $3(\mathbf{n L} 1=3, \mathbf{n L} 2=5)$

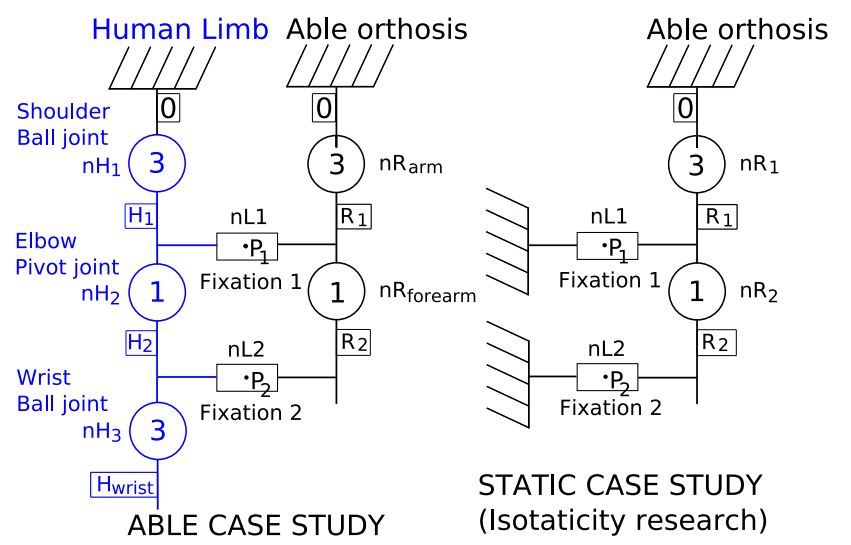

Fig. 9. Schematic of the ABLE and human arm coupling

Four different choices are thus possible for $\mathbf{n L}_{1}$ each of them leading in one possible choice for $\mathbf{n L}_{2}$ thanks to Eq.5. The different solutions are listed below in the table I: Several

\begin{tabular}{||c|c|c|c|c|c|}
\hline & $\mathbf{n L} 2=2$ & $\mathbf{n L} 2=3$ & $\mathbf{n L} 2=4$ & $\mathbf{n L} 2=5$ & $\mathbf{n L}=6$ \\
\hline $\mathbf{n L} 1=3$ & & & & OK & $\mathrm{X}$ \\
\hline $\mathbf{n L} 1=4$ & & & OK & $\mathrm{X}$ & $\mathrm{X}$ \\
\hline $\mathbf{n L} 1=5$ & & $\mathrm{OK}$ & $\mathrm{X}$ & $\mathrm{X}$ & $\mathrm{X}$ \\
\hline $\mathbf{n L} 1=6$ & $\mathrm{OK}$ & $\mathrm{X}$ & $\mathrm{X}$ & $\mathrm{X}$ & $\mathrm{X}$ \\
\hline
\end{tabular}

TABLE I

CATALOG OF SOLUTiONS

passive fixations kinematics solutions can be seen on Figure 10. Choosing 6 DOF on a fixation leads to the liberation of the segment, and so on a loss of control. Whatever, rule 3 advises us to have several fixations. Liberating 5 or $6 \mathrm{DOF}$ at this fixation point leads to a limited control of the arm coordination and to an orthosis only connected and comanipulated by the forearm. This should have an effect on the force concentration leading to pain. Ideally for the fixation of an human limb, considering our design paradigm (the 4 rules enunciated before), we should choose $\mathbf{n} \mathbf{L}_{i} \geq 4$, in order to liberate the rotation to prevent moment appearance (according to rule 1 ) and the translation along the member axis (according to rule 2). We thus choose $\mathbf{n L}_{1}=\mathbf{n} \mathbf{L}_{2}=4$.

Applying rule 4 leads us to put in place the fixation on specific surfaces with a reduce muscular mass between skin and bone allowing an important contact stiffness and a limited diameter variation during flexion-extension cycles. First fixation will be placed on the arm near the elbow joint and the other one on the forearm near the wrist joint. In order to limit the crushing, the fixations will be not placed too near of the limb joint, because skin sensitivity is increased near these areas [17]. Note that force control problem specificity is as follows:

- a 4 actuated DOF robotic system to control.

- an human arm with 7 or 9 actuated DOF (depending on the consideration of the scapula mobility), which is similar to an 4 actuated DOF system when observed through the passive joint fixations we add.

- a 6-axis force/torque (F/T) sensor at the two fixation point $\mathbf{P} i$.

Adding a ball joint and a slide at the two fixation points $\mathbf{P}_{1}$ and $\mathbf{P}_{1}$ between force sensor and fixation area, lead to a reduction of the external force/torque matrix column that evolve from a $(12 \times 1)$ to a $(4 \times 1)$ dimension (only two forces can be generated at each connexion point) and so on to a virtual reduction of $\mathbf{J}^{\mathrm{T}}$ making the system force controllable and stable. We can thus write that

$$
\Gamma=\left[\mathbf{J}_{1 \mathrm{~T}} \mathbf{J}^{\mathrm{T}}{ }_{2}\right] \cdot\left(\begin{array}{c}
\mathbf{F}_{\text {ext } \rightarrow 1} \\
\mathbf{M}_{\text {ext } \rightarrow 1}\left(\mathbf{P}_{1}\right) \\
\mathbf{F}_{\text {ext } \rightarrow 2} \\
\mathbf{M}_{\text {ext } \rightarrow 2}\left(\mathbf{P}_{2}\right)
\end{array}\right)=\mathbf{J}_{R}{ }^{\mathrm{T}} \cdot\left(\begin{array}{c}
\mathbf{F} \mathbf{x}_{\text {ext } \rightarrow 1} \\
\mathbf{F} \mathbf{y}_{\text {ext } \rightarrow 1} \\
\mathbf{F} \mathbf{x}_{\text {ext } \rightarrow 2} \\
\mathbf{F} \mathbf{y}_{\text {ext } \rightarrow 2}
\end{array}\right)
$$

\section{Fixations realization}

To free four rotations at every fixation point, we firstly choose to add a ball joint with a hole on a linear guide. This first solution has quickly been improved. Due to the space and weight limitations (and to avoid interaction with the trunk) we transform the standard ball-joint into a simplified yet fully 
functional ball-joint mechanism eliminating the discomforting strapping. We also place a linear guide inside the ball-joint

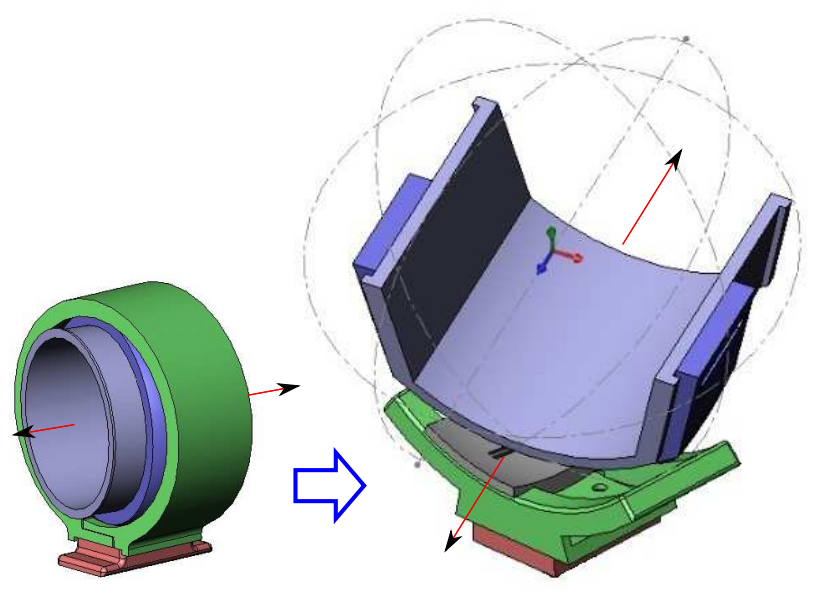

Fig. 11. Fixation design

mechanism to prevent angle discrepancies which may lead to force projection along the main limb segment axis. Using the same model, different opening sizes adapted to every subject physiology. In addition to different dimensions, an inflatable bag can be added inside the ball joint to allow a better adaptation to variant morphology.

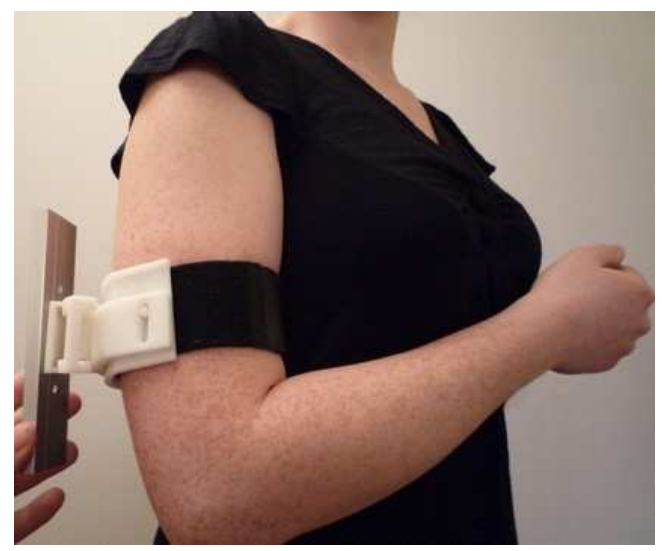

Fig. 12. View of the arm fixation in situation

\section{CONCLUSION}

In this paper, we presented a methodology aimed at designing the fixations with specific kinematics for attaching an orthosis to a human limb. We suggested an iterative tool and a set of design rules allowing a simple resolution of the hyperstaticity problem when robots and human limbs are coupled through multiple connection points. The provided solution not only avoids hyperstaticity but also resolves the problem that arrises when faced with a large variation of human geometry. Force control models were also provided.

The future work will be focused on deploying a multi-contact force control on the ABLE orthosis. The orthosis would be fitted with the F/T sensors with the chosen (mentioned) passive
DOF fixations to experimentally evaluate the viability and stability of our design approach.

\section{ACKNOWLEDGMENTS}

The authors gratefully acknowledge the contribution of National Research Organization and reviewers' comments.

\section{REFERENCES}

[1] N. Jarrasse, J. Robertson, P. Garrec, J. Paik, V. Pasqui, Y. Perrot, A. Roby-Brami, D. Wang, and G. Morel. Design and acceptability assessment of a new reversible orthosis. Intelligent Robots and Systems, 2008. IROS 2008. IEEE/RSJ International Conference on, pages 19331939, Sept. 2008.

[2] H.I. Krebs, N. Hogan, M.L. Aisen, and B.T. Volpe. Robot-aided neurorehabilitation. IEEE transactions on rehabilitation engineering., 6, Mar 1998.

[3] P. Garrec, JP. Martins, and JP. Friconneau. A new technology for portable exoskeletons. AMSE2004, 2004.

[4] Shimada Yoichi and Terayama Yukio. Embedded microprocessor system for the transtibial active prosthesis control. IEIC Technical Report, pages 17-20, 2006.

[5] J.C. Perry, J. Rosen, and S. Burns. Exoskeletons for gait assistance and training of the motor-impaired. Mechatronics, IEEE/ASME Transactions on, Aug 2007.

[6] A. Gupta and M.K. O'Malley. Design of a haptic arm exoskeleton for training and rehabilitation. Mechatronics, IEEE/ASME Transactions on, June 2006.

[7] A.M. Dollar and H. Herr. Development of a wearable robot for assisting carpentry workers. Robotics, IEEE Transactions on, 24, Feb. 2008.

[8] Junpei NAITO, Goro OBINATA, Atsushi NAKAYAMA, and Kazunori HASE. Lower extremity exoskeletons and active orthoses: Challenges and state-of-the-art. International Journal of Advanced Robotic System, 2006.

[9] Jose L. Pons. Wearable Robots: Biomechatronic Exoskeletons. 2008.

[10] P. Culmer, A. Jackson, R. Richardson, B. Bhakta, M. Levesley, and A. Cozens. Development of a dual robotic system for upper-limb stroke rehabilitation. Rehabilitation Robotics, 2005. ICORR 2005. 9th International Conference on, 2005.

[11] Scott SH and Winter DA. Biomechanical model of the human foot: kinematics and kinetics during the stance phase of walking. J Biomech., 21993.

[12] Van der helm, Veeger, and Pronk HG. M. Geometry parameters for musculoskeletal modelling of the shoulder system. Journal of biomechanics, June 1992.

[13] A. Schiele and F.C.T. van der Helm. Kinematic design to improve ergonomics in human machine interaction. Neural Systems and Rehabilitation Engineering, IEEE Transactions on, 14(4):456-469, Dec. 2006.

[14] A. Schiele. An explicit model to predict and interpret constraint force creation in phri with exoskeletons. Robotics and Automation, 2008. ICRA 2008. IEEE International Conference on, pages 1324-1330, May 2008.

[15] S.J. Housman, Vu Le, T. Rahman, R.J. Sanchez, and D.J. Reinkensmeyer. Arm-training with t-wrex after chronic stroke: Preliminary results of a randomized controlled trial. Rehabilitation Robotics, 2007. ICORR 2007. IEEE 10th International Conference on, June 2007.

[16] P. Garrec, J.P. Friconneau, Y. Measson, and Y. Perrot. Able, an innovative transparent exoskeleton for the upper-limb. Intelligent Robots and Systems, 2008. IROS 2008. IEEE/RSJ International Conference on, pages 1483-1488, Sept. 2008.

[17] Bystrom S, Hall C, Welander T, and Kilbom A. Clinical disorders and pressure-pain threshold of the forearm and hand among automobile... Journal of Hand Surgery, 20, 1995. 